\title{
Application of Filippov method for the analysis of subharmonic instability in dc-dc converters
}

\author{
Damian Giaouris ${ }^{1, *, \dagger}$, Somnath Maity ${ }^{2}$, Soumitro Banerjee ${ }^{2}$, Volker Pickert ${ }^{1}$ \\ and Bashar Zahawi ${ }^{1}$ \\ ${ }^{1}$ School of Electrical, Electronic and Computer Engineering, Newcastle University, \\ Newcastle upon Tyne NE1 7RU, U.K. \\ ${ }^{2}$ Department of Electrical Engineering, Indian Institute of Technology, Kharagpur 721302, India
}

\begin{abstract}
SUMMARY
We propose a method of estimating the fast-scale stability margin of dc-dc converters based on Filippov's theory-originally developed for mechanical systems with impacts and stick-slip motion. In this method one calculates the state transition matrix over a complete clock cycle, and the eigenvalues of this matrix indicate the stability margin. Important components of this matrix are the state transition matrices across the switching events, called saltation matrices. We applied this method to estimate the stability margins of a few commonly used converter and control schemes. Finally, we show that the form of the saltation matrix suggests new control strategies to increase the stability margin, which we experimentally demonstrate using a voltage-mode-controlled buck converter. Copyright (C) 2008 John Wiley \& Sons, Ltd.
\end{abstract}

Received 16 November 2007; Revised 14 April 2008; Accepted 27 April 2008

KEY WORDS: dc-dc converter; fast-scale stability; subharmonic oscillation; pulse width modulation; switching control

\section{INTRODUCTION}

It is considered desirable to operate dc-dc converters with constant switching frequency equal to that of the external clock. The stability of this periodic mode of operation may, however, be lost due to variation of parameters such as the input voltage or the load, resulting in subharmonic oscillations $[1,2]$. In order to avoid the occurrence of subharmonic oscillations in practical converters, it is normal practice to specify the range of external parameters within which the converter will operate reliably in steady state.

\footnotetext{
${ }^{*}$ Correspondence to: Damian Giaouris, School of Electrical, Electronic and Computer Engineering, Newcastle University, Newcastle upon Tyne NE1 7RU, U.K.

†E-mail: damian.giaouris@ncl.ac.uk
} 
The internal parameters of the converter (the inductance, capacitance, clock speed, etc.) are chosen on the basis of the averaged model [3,4] to obtain the desired voltage ratio, power throughput, transient behavior, etc. Although this is effective in ensuring stability and the desired transient performance at slow timescale, it provides no information regarding stability of the cycleto-cycle fast timescale dynamics. It is therefore necessary to estimate the fast-scale stability margin at the clock speed and thus, the range of the external parameters that will ensure periodic operation without the onset of subharmonic oscillations.

The sampled-data model was developed by Verghese et al. [5] to address this problem. This modeling procedure makes discrete observation of the state at every clock instant (in some literature the sampled-data model is also referred to as the Poincare map [6,7]). The problem of the stability of the trajectory in the state space then reduces to that of the stability of the fixed point of the sampled-data model $[8,9]$. Other methods have also been applied such as the trajectory sensitivity [10], which yields a discrete-algebraic-differential model with various switching/jump conditions. An auxiliary vector has been proposed in [11] which again produces similar results as the Poincare map and the trajectory sensitivity approach. Such techniques have also been applied to analyze more complicated systems such as the Ćuk converter [12], power factor correctors [13, 14], parallel connected converters [15] and fuzzy logic controllers [16]. However, even though these approaches are conceptually simple, they have not found widespread acceptance among mainstream power electronic practitioners-probably because of the mathematical complexity involved.

At present the fast-scale stability is mostly checked by simulation or experimentation over a large parameter range. In most cases, the components are overdesigned, thus practically eliminating the problem of subharmonics. However, with the push toward miniaturization, and the advent of on-chip power supplies, one is constrained to design the components in a minimum possible size. This has provided the renewed motivation for developing a useful and simple technique for estimating the fast-scale stability margin.

In this paper we present an alternative method of estimating the fast-scale stability margin of dc-dc converters. It achieves the same objective as the sampled-data model, but in a more straightforward way. The technique is based on the theory originally developed by Floquet [17] to study the stability of periodic orbits, and then extended by Aizerman, Gantmakher and Filippov to analyze impacting motion and stick-slip oscillations in mechanical switching systems $[18,19]$. The purpose of this paper is to show that this theoretical structure is very suitable to the study of fast-scale stability of dc-dc converters which belong to the same class of switching dynamical systems as the mechanical systems for which the theory was originally developed.

However, in applying this method to dc-dc converters, some specific issues need to be addressed. We illustrate this using a number of commonly used converters and control strategies. First, we consider the buck converter with voltage mode control operating in the continuous conduction mode as well as in the discontinuous conduction mode (DCM). We demonstrate the specific problem in applying the technique to PWM of type-1, and the way to overcome it. Then we study the stability of the boost converter which is normally used with peak or average current mode control.

It is interesting to note here that all the available methods such as sampled-data modeling, trajectory sensitivity analysis, etc. are based on three basic steps: A local approximation, the solution of a matrix differential equation and the use of a stability criterion [20]. Since all the techniques describe the stability of periodic orbits, they produce the same final matrix (given various names such as the Jacobian of the Poincare map or monodromy matrix [21]) and the same eigenvalues (also called Floquet multipliers). For example, Equations (64) in [10], (5.49) in [6] and (7.64) in [22] are very similar. Out of all the mathematically equivalent formalisms, the method 
presented in this paper is more convenient because it studies the effects of each switching on the stability of the system separately.

The proposed technique also offers a powerful strategy for controlling the instability that results in subharmonic oscillations. In the current-mode-controlled converters, a compensation ramp is generally used to control the onset of subharmonic oscillations. We show that similar methods can be evolved for controlling subharmonic instabilities and the onset of slow-scale sinusoidal oscillations in other converters and PWM strategies. The basic concept here is to employ a supervising controller (using system variables such as current and voltage ripple as feedback), which alters specific saltation matrix parameters to stabilize the periodic solution without greatly altering its location in the state space.

\section{AN OUTLINE OF THE FILIPPOV METHOD}

In a dc-dc converter one is interested in the stability of a periodic orbit that starts at a specific state at a clock instant and returns to the same state at the end of the clock period. The stability of such a periodic orbit can be understood in terms of the evolution of perturbation. If the initial condition is perturbed and the solution converges back to the orbit, then the orbit is stable. The stability margin can be assessed from the rate of convergence.

Suppose a given system has an initial condition $\mathbf{x}\left(t_{0}\right)$ at time $t_{0}$, and we perturb it to $\overline{\mathbf{x}}\left(t_{0}\right)$ such that the perturbation is $\Delta \mathbf{x}\left(t_{0}\right)=\mathbf{x}\left(t_{0}\right)-\overline{\mathbf{x}}\left(t_{0}\right)$. If the original trajectory and the perturbed trajectory evolve for a time $t$, the perturbation at the end of the period can be related to the initial perturbation by

$$
\Delta \mathbf{x}(t)=\mathbf{\Phi} \Delta \mathbf{x}\left(t_{0}\right)
$$

where $\boldsymbol{\Phi}$ is the state transition matrix, which is a function of the initial state, the initial time and the final time. It is known that in linear time-invariant (LTI) systems, the state transition matrix is given by the matrix exponential

$$
\boldsymbol{\Phi}=\mathrm{e}^{\mathbf{A}\left(t-t_{0}\right)}
$$

where $\mathbf{A}$ is the state matrix that appears in the state equation $\dot{\mathbf{x}}=\mathbf{A x}+\mathbf{B u}$.

Now, for any dc-dc converter, the state evolves through subsystems (ON state of the switch, OFF state of the switch, etc.) that are LTI. Therefore, for the evolution through each subsystem, the state transition matrix can be obtained by the above formula (if the initial time, the final time and the initial condition are known), and can be explicitly evaluated using MATLAB's expm function.

Suppose the state evolves from the instant $t_{A}$ to the instant $t_{B}$, and the state transition matrix for that period is $\boldsymbol{\Phi}_{1}$; then it evolves from the instant $t_{B}$ to $t_{C}$, and the state transition matrix in that interval is $\boldsymbol{\Phi}_{2}$. It is known that if the evolution from $t_{A}$ to $t_{C}$ is smooth (everywhere differentiable), then the state transition matrix from $t_{A}$ to $t_{C}$ is simply the product of the two matrices $\boldsymbol{\Phi}_{2} \boldsymbol{\Phi}_{1}$. However, if a switching occurs at point $B$, the evolution becomes nonsmooth at that point since the governing equations before and after the event are different. Aizerman and Gantmakher [18] and Filippov [19] showed that in such a situation one has to additionally consider the evolution of the perturbation across the switching event. They derived the form of the state transition matrix $\mathbf{S}$ that relates the perturbation just after the switching event to that just before

$$
\Delta \mathbf{x}\left(t_{B+}\right)=\mathbf{S} \Delta \mathbf{x}\left(t_{B-}\right)
$$


which is called the 'jump matrix' [10] or 'saltation matrix' [22] or 'updating matrix' [23, 24]. It was shown $[22,25]$ that the saltation matrix can be expressed as

$$
\mathbf{S}=\mathbf{I}+\frac{\left(\mathbf{f}_{+}-\mathbf{f}_{-}\right) \mathbf{n}^{\mathrm{T}}}{\mathbf{n}^{\mathrm{T}} \mathbf{f}_{-}+\partial h / \partial t}
$$

where $\mathbf{I}$ is the identity matrix of the same order as the number of state variables, $h(\mathbf{x}, t)=0$ represents the switching condition (a surface in the state space of the system), $\mathbf{n}$ is the vector normal to the switching surface and $\mathbf{n}^{\mathrm{T}}$ is its transpose. $\mathbf{f}_{-}$represents the right-hand side of the differential equations before the switching occurred and $\mathbf{f}_{+}$represents the right-hand side of the differential equations after the switching. From this expression the saltation matrix can be evaluated for each switching event.

Since we are interested in the stability of the periodic orbit exhibited by the converters, we need to calculate the state transition matrix over a whole clock cycle. This matrix is called the monodromy matrix. If the switch is $\mathrm{ON}$ at the beginning of the clock cycle from time 0 till time $d T$ (where $d$ is the duty ratio), and the switch remains OFF from time $d T$ till time $T$ (the clock period), then the monodromy matrix is expressed as

$$
\boldsymbol{\Phi}_{\text {cycle }}(T, 0)=\mathbf{S}_{2} \times \boldsymbol{\Phi}_{\text {off }}(T, d T) \times \mathbf{S}_{1} \times \boldsymbol{\Phi}_{\text {on }}(d T, 0)
$$

where $\mathbf{S}_{1}$ is the saltation matrix related to the first switching event, and $\mathbf{S}_{2}$ is that related to the second switching event.

The monodromy matrix then relates the perturbation at the end of the clock period to that at the beginning:

$$
\Delta \mathbf{x}(T)=\Phi(T, 0) \Delta \mathbf{x}(0)
$$

If the moduli of all the eigenvalues of the monodromy matrix are less than unity (i.e. if all the eigenvalues are inside the unit circle), perturbations will die down and the system will be stable. The eigenvalues of the monodromy matrix are also called the Floquet multipliers, whose distance from the unit circle indicates the stability margin.

\section{THE VOLTAGE-MODE-CONTROLLED BUCK CONVERTER}

We now illustrate the method with reference to the voltage-mode-controlled buck converter, whose schematic diagram is shown in Figure 1. The switching signal can be generated (see Figure 2) either by comparing the control voltage at the beginning of a clock period with a ramp waveform (type-1 PWM or PWM-1), or by comparing the instantaneous value of the control voltage with a ramp waveform (type-2 PWM or PWM-2). We first consider a PWM-2 control that is more prevalent in analog implementations.

\subsection{Continuous conduction mode}

When the switch is $\mathrm{ON}$, the state equations are

$$
\frac{\mathrm{d} v}{\mathrm{~d} t}=-\frac{v}{R C}+\frac{i}{C}, \quad \frac{\mathrm{d} i}{\mathrm{~d} t}=-\frac{v}{L}+\frac{V_{\text {in }}}{L}, \quad \frac{\mathrm{d} v_{i}}{\mathrm{~d} t}=K_{I}\left(v-V_{\text {ref }}\right)
$$




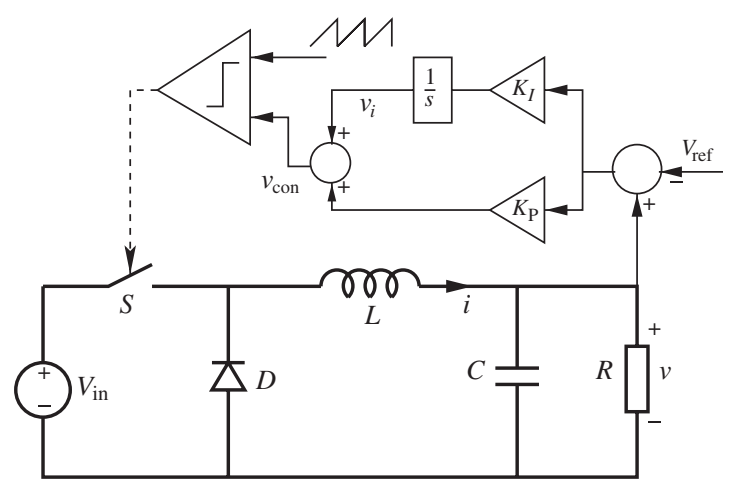

Figure 1. The schematic diagram of the voltage-mode-controlled buck converter.

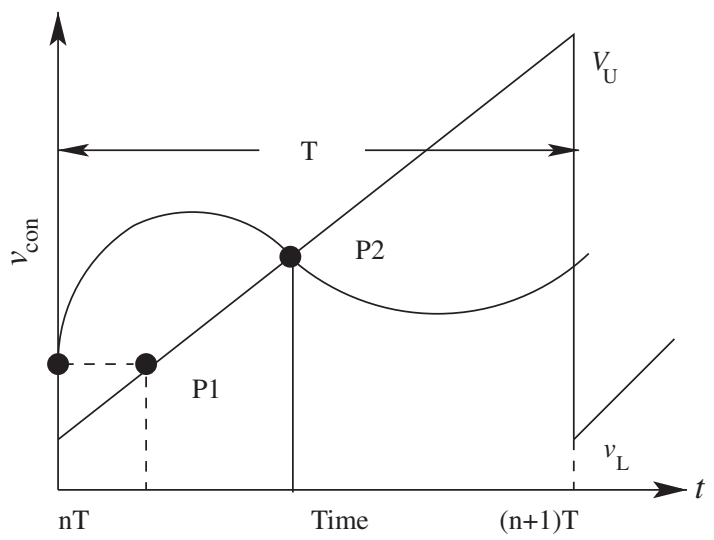

Figure 2. The difference between type-1 and type-2 PWM switchings. In PWM-1, the switching occurs at point $P 1$ whereas in PWM-2 it occurs at point $P 2$.

When the switch is off, the equations are

$$
\frac{\mathrm{d} v}{\mathrm{~d} t}=-\frac{v}{R C}+\frac{i}{C}, \quad \frac{\mathrm{d} i}{\mathrm{~d} t}=-\frac{v}{L}, \quad \frac{\mathrm{d} v_{i}}{\mathrm{~d} t}=K_{I}\left(v-V_{\mathrm{ref}}\right)
$$

One peculiarity of this system is that the output of the integrator (the state $v_{i}$ ) does not appear in the right-hand side of the system equations, and plays role only in deciding the switching condition. That is why any perturbation in that variable does not affect any of the states. The integrator-which is necessary to avoid noise feedback-acts as a slow first-order low-pass filter that is effectively isolated from the rest of system dynamics. We shall see later that this gives rise to one eigenvalue very close to +1 , which does not change with the variation of the parameters.

For the sake of compact notation, we express the equations in terms of the state vector $\mathbf{x}$, where $x_{1}$ is the capacitor voltage $v, x_{2}$ the inductor current $i, x_{3}$ the output of the integrator in the 
feedback loop $v_{i}$, and the state equations are expressed as

$$
\dot{\mathbf{x}}(t)= \begin{cases}\mathbf{f}_{\text {off }}(\mathbf{x}, t)=\mathbf{A}_{\text {off }} \mathbf{x}+\mathbf{B}_{\text {off }} \mathbf{u}, & S \text { is off } \\ \mathbf{f}_{\text {on }}(\mathbf{x}, t)=\mathbf{A}_{\text {on }} \mathbf{x}+\mathbf{B}_{\text {on }} \mathbf{u}, & S \text { is on }\end{cases}
$$

For the buck converter,

$$
\mathbf{A}_{\text {off }}=\mathbf{A}_{\text {on }}=\mathbf{A}=\left[\begin{array}{ccc}
-1 / R C & 1 / C & 0 \\
-1 / L & 0 & 0 \\
K_{I} & 0 & 0
\end{array}\right]
$$

and

$$
\mathbf{B}_{\text {off }}=\left[\begin{array}{c}
0 \\
0 \\
-K_{I}
\end{array}\right], \quad \mathbf{B}_{\text {on }}=\left[\begin{array}{c}
0 \\
1 / L \\
-K_{I}
\end{array}\right], \quad \mathbf{u}=\left[\begin{array}{c}
0 \\
V_{\text {in }} \\
V_{\text {ref }}
\end{array}\right]
$$

The control voltage obtained from the output of the PI controller is

$$
v_{\text {con }}=K_{P}\left(x_{1}-V_{\text {ref }}\right)+x_{3}
$$

The switch conducts when the output of the PI controller is less than $v_{\text {ramp }}=V_{L}+\left(V_{U}-\right.$ $\left.V_{L}\right)(t / T \bmod 1)$.

Suppose, on the basis of the customer specification and the averaged model, the designer has chosen the parameter values $V_{\text {ref }}=11.3 \mathrm{~V}, L=20 \mathrm{mH}, C=47 \mu \mathrm{F}, K_{P}=8, K_{I}=10, T=1 / 2500 \mathrm{~s}$, and the ramp signal varies from 3.8 to $8.2 \mathrm{~V}$. Using PSPICE or SIMULINK simulation, one can easily check that if the external parameters are $R=22 \Omega$ and $V_{\text {in }}=25 \mathrm{~V}$, the converter operates in the stable periodic mode. The experimentally obtained waveforms in this mode are shown in Figure 3. The question is, how stable is this behavior?

To probe this question, we note that the OFF period lasts for $(1-d) T$ seconds, and the ON period lasts for $d T$ seconds. Therefore, the state transition matrices for these two intervals are

$$
\boldsymbol{\Phi}_{\text {off }}=\mathrm{e}^{\mathbf{A}(1-d) T} \quad \text { and } \quad \boldsymbol{\Phi}_{\text {on }}=\mathrm{e}^{\mathbf{A} d T}
$$

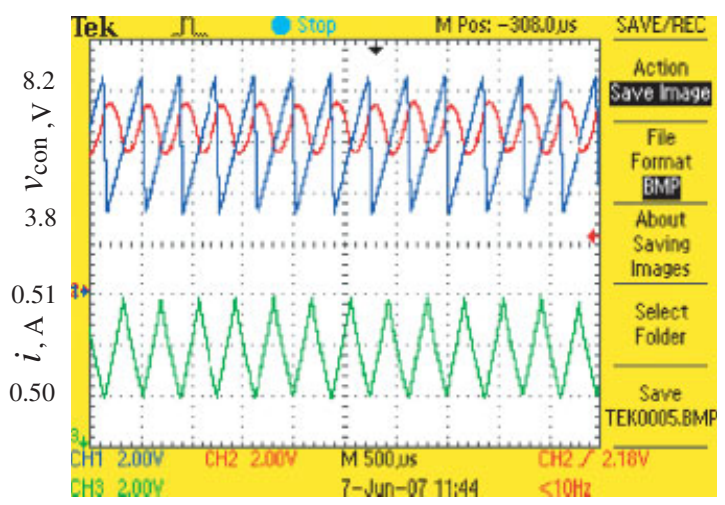

Figure 3. Experimental waveforms for the voltage and current ripples for $R=22 \Omega$ and $V_{\text {in }}=25 \mathrm{~V}$. 
In order to evaluate these, one needs to know the duty ratio. This can be obtained following $[5,20,26,27]$ using the Newton-Raphson method. Alternatively, one can use any standard simulator to obtain the stable behavior, from which the information about $d$ can be extracted. Thus, we assume that the steady-state value of the duty ratio $d$ is known. For the chosen set of parameters, the duty ratio was found to be $d=0.5480$. Substituting the parameter values, we obtain

$$
\boldsymbol{\Phi}_{\text {off }}=\left[\begin{array}{ccc}
0.7868 & 4.1670 & 0 \\
-0.01 & 0.9763 & 0 \\
0.0020 & 0.0047 & 1
\end{array}\right], \quad \boldsymbol{\Phi}_{\text {on }}=\left[\begin{array}{ccc}
0.8241 & 3.5088 & 0 \\
-0.008 & 0.9836 & 0 \\
0.0016 & 0.0033 & 1
\end{array}\right]
$$

Now we need to calculate the saltation matrix to account for the switching. Within one period of the clock, the switching condition can be defined as $h(\mathbf{x}, t)=0$, where

$$
h(\mathbf{x}, t)=K_{P}\left(x_{1}-V_{\text {ref }}\right)+x_{3}-V_{L}-\left(V_{U}-V_{L}\right) t / T
$$

Hence, its normal vector and the rate of change are given by

$$
\mathbf{n}=\left[\begin{array}{c}
\partial h / \partial x_{1} \\
\partial h / \partial x_{2} \\
\partial h / \partial x_{3}
\end{array}\right]=\left[\begin{array}{c}
K_{P} \\
0 \\
1
\end{array}\right] \text { and } \frac{\partial h}{\partial t}=-\frac{\left(V_{U}-V_{L}\right)}{T}
$$

Now, since

$$
\mathbf{f}_{\mathrm{on}}(\mathbf{x}, t)=\left[\begin{array}{c}
\frac{R x_{2}-x_{1}}{R C} \\
\frac{V_{\mathrm{in}}-x_{1}}{L} \\
K_{I}\left(x_{1}-V_{\mathrm{ref}}\right)
\end{array}\right], \quad \mathbf{f}_{\mathrm{off}}(\mathbf{x}, t)=\left[\begin{array}{c}
\frac{R x_{2}-x_{1}}{R C} \\
\frac{-x_{1}}{L} \\
K_{I}\left(x_{1}-V_{\mathrm{ref}}\right)
\end{array}\right]
$$

we have

$$
\left(\mathbf{f}_{\mathrm{on}}-\mathbf{f}_{\mathrm{off}}\right) \mathbf{n}^{\mathrm{T}}=\left[\begin{array}{c}
0 \\
V_{\mathrm{in}} / L \\
0
\end{array}\right]\left[\begin{array}{lll}
K_{P} & 0 & 1
\end{array}\right]=\left[\begin{array}{ccc}
0 & 0 & 0 \\
\frac{K_{P} V_{\text {in }}}{L} & 0 & \frac{V_{\text {in }}}{L} \\
0 & 0 & 0
\end{array}\right]
$$

and

$$
\begin{aligned}
\mathbf{n}^{\mathrm{T}} \mathbf{f}_{\mathrm{off}} & =\left[\begin{array}{lll}
K_{P} & 0 & 1
\end{array}\right]\left[\begin{array}{c}
\frac{R x_{2}-x_{1}}{R C} \\
\frac{-x_{1}}{L} \\
K_{I}\left(x_{1}-V_{\text {ref }}\right)
\end{array}\right] \\
& =K_{P} \frac{R x_{2}-x_{1}}{R C}+K_{I}\left(x_{1}-V_{\text {ref }}\right)
\end{aligned}
$$


Substituting these into the expression for the saltation matrix (1), we obtain

$$
\mathbf{S}_{1}=\left[\begin{array}{ccc}
1 & 0 & 0 \\
s_{21} & 1 & s_{23} \\
0 & 0 & 1
\end{array}\right]
$$

where

$$
\begin{aligned}
& s_{21}=\frac{K_{P} V_{\text {in }} / L}{K_{P} \frac{R x_{2}-x_{1}}{R C}+K_{I}\left(x_{1}-V_{\text {ref }}\right)-\frac{V_{U}-V_{L}}{T}} \\
& s_{23}=\frac{V_{\text {in }} / L}{K_{P} \frac{R x_{2}-x_{1}}{R C}+K_{I}\left(x_{1}-V_{\text {ref }}\right)-\frac{V_{U}-V_{L}}{T}}
\end{aligned}
$$

which are to be evaluated at the switching instant $t=(1-d) T$. Substituting the values, we get the saltation matrix as

$$
\mathbf{S}_{1}=\left[\begin{array}{ccc}
1 & 0 & 0 \\
-0.4656 & 1 & -0.0582 \\
0 & 0 & 1
\end{array}\right]
$$

The second saltation matrix $\mathbf{S}_{2}$ related to the switching from the ON state to the OFF state at the end of the clock cycle turns out to be an identity matrix, because the falling edge of the ramp causes the $\partial h / \partial t$ term in Equation (1) to be infinity.

Hence, the state transition matrix over the complete cycle (the monodromy matrix) is calculated as

$$
\boldsymbol{\Phi}_{\text {cycle }}=\mathbf{S}_{2} \boldsymbol{\Phi}_{\text {on }} \mathbf{S}_{1} \boldsymbol{\Phi}_{\text {off }}=\left[\begin{array}{ccc}
-0.6717 & 0.0514 & -0.2042 \\
-0.3766 & -0.9827 & -0.0572 \\
0.0020 & 0.0085 & 0.9998
\end{array}\right]
$$

The eigenvalues of the monodromy matrix were found to be $-0.76029,-0.89376$ and 0.99951 . As expected, one eigenvalue comes very close to +1 , which is actually contributed by the integrator of the controller. As we have already noted, this eigenvalue does not contribute to the system's stability, and has to be ignored while estimating the stability margin. ${ }^{\ddagger}$ We see that the second eigenvalue is reasonably close to -1 and hence the system has a poor stability margin - a fact that was in no way evident from circuit simulation or the experimental waveform presented in Figure 3.

This prediction is confirmed as the input voltage is increased from 25 to $27 \mathrm{~V}$, and experiment shows that period-2 subharmonic oscillation sets in (see Figure 4, where the same state repeats

\footnotetext{
${ }^{\ddagger}$ In fact, with the same duty ratio, if the saltation matrix calculation is done using only two variables, the eigenvalues of the monodromy matrix are obtained as -0.76021 and -0.89343 . This shows that very little error is incurred if the stability calculation is simplified by considering $2 \times 2$ matrices.
} 


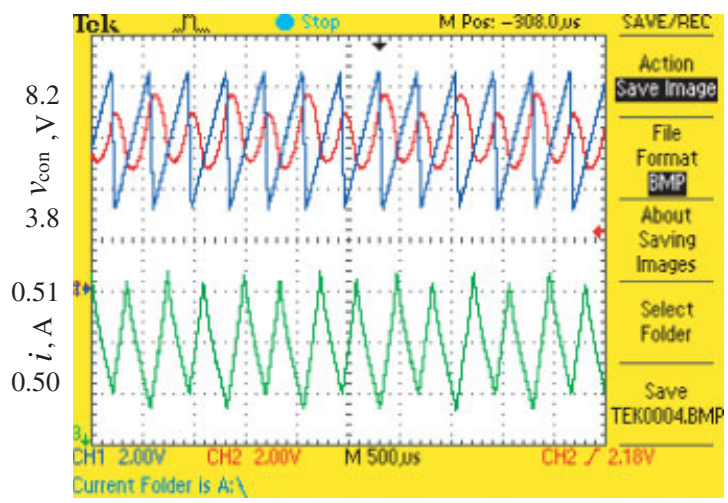

Figure 4. The voltage and current ripples at $R=22 \Omega$ and $V_{\text {in }}=27 \mathrm{~V}$ observed in the experiment.

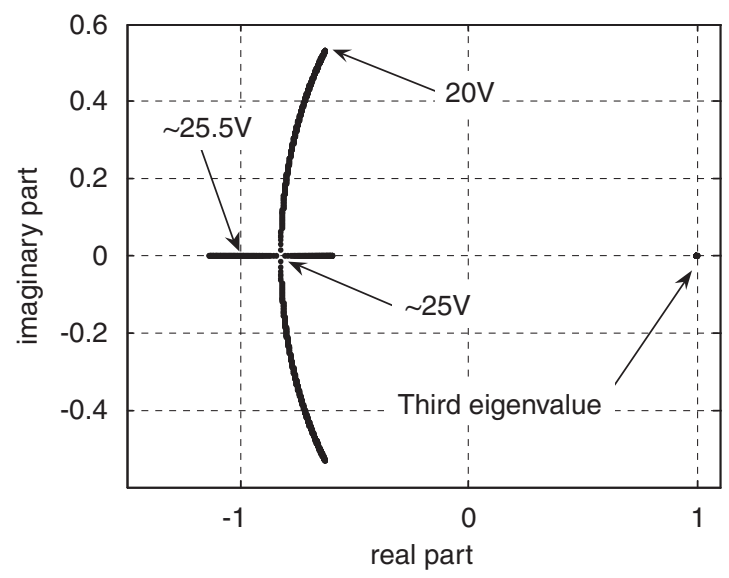

Figure 5. The locus of the eigenvalues of the monodromy matrix.

every two clock periods). This period-doubling instability has been reported in numerous studies on the buck converter [28-30].

By our method one can obtain the locus of the eigenvalues as a parameter is varied, which is shown in Figure 5. It shows that the eigenvalues are complex conjugate at $V_{\text {in }}=20 \mathrm{~V}$, which move on a circle toward each other as the parameter is increased, and become real at $V_{\text {in }} \approx 25 \mathrm{~V}$. Thereafter, they move away from each other, and at $V_{\mathrm{in}}=25.5 \mathrm{~V}$ one of the eigenvalues move out of the unit circle. Thus, one can find the exact parameter value at which the fast-scale stability is lost (in this case $V_{\text {in }}=25.5 \mathrm{~V}$ ). It is known from bifurcation theory that a period-2 subharmonic occurs if one of the eigenvalues equals -1 at the point of instability, and this is what we see in Figure 4. It is notable that the third eigenvalue does not shift with the change in parameter.

By the technique introduced in this paper, one can also obtain the range of external parameters for which stable periodic operation is obtained (Figure 6). Such a diagram helps in setting the design limits, as it shows that the safe range of $V_{\text {in }}$ depends on the load, and the safe range of $R$ depends on the input voltage. 


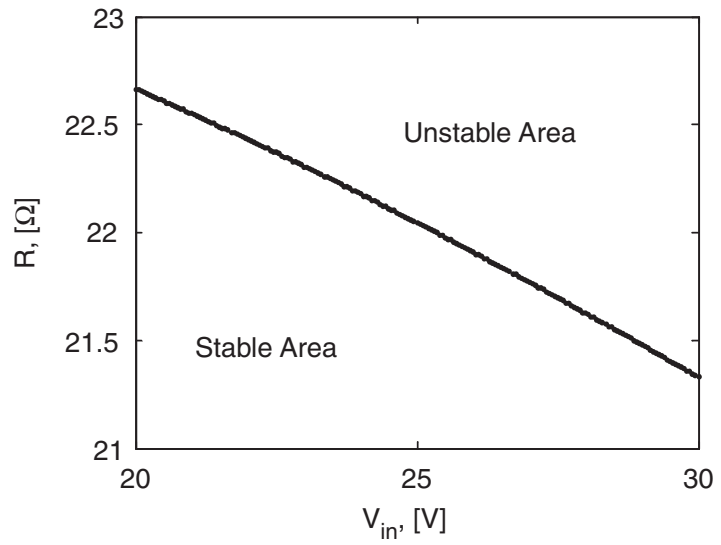

Figure 6. The parameter space map showing the stable region.

\subsection{Discontinuous conduction mode}

Low rating power converters are normally operated in DCM [31], and in that case one has to consider the state equation when $i=0$ in addition to those appearing in Equation (3). Since we have seen in the last section that the integrator in the feedback path only adds an extra dimension whose associated eigenvalue is inconsequential in deciding the fast-scale stability of the system, we ignore that extra variable in this section. However, the integrator has effect in deciding the duty ratio, and hence all the three variables are considered when computing the instants of switching.

The state equations are

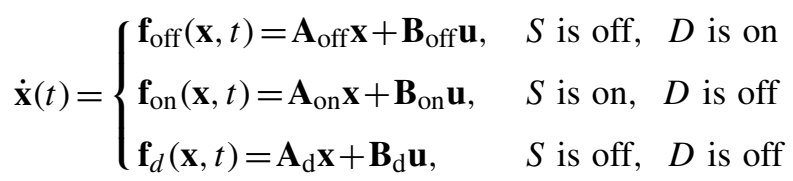

where

$$
\begin{aligned}
& \mathbf{A}_{\text {off }}=\mathbf{A}_{\text {on }}=\mathbf{A}=\left[\begin{array}{cc}
-1 / R C & 1 / C \\
-1 / L & 0
\end{array}\right], \quad \mathbf{A}_{\mathrm{d}}=\left[\begin{array}{cc}
-1 / R C & 0 \\
0 & 0
\end{array}\right] \\
& \mathbf{B}_{\text {off }}=\left[\begin{array}{l}
0 \\
0
\end{array}\right], \quad \mathbf{B}_{\text {on }}=\left[\begin{array}{c}
0 \\
1 / L
\end{array}\right], \quad \mathbf{B}_{\mathrm{d}}=\left[\begin{array}{l}
0 \\
0
\end{array}\right], \quad \mathbf{u}=\left[\begin{array}{c}
0 \\
V_{\text {in }}
\end{array}\right]
\end{aligned}
$$

In this case, the system starts the clock cycle in the ON state which continues for a period of $d_{1} T$, the switch turns off when $v_{\text {con }}<v_{\text {ramp }}$ at $d_{2} T$, and the zero-current phase continues for a period $\left(1-d_{2}\right) T$. One can obtain $d_{1}, d_{2}$ and the values of the state at the switching instants from simulation or by the Newton-Raphson method following [5].

The state transition matrix over the complete cycle will be composed of the state transition matrices over the three phases of evolution, and three saltation matrices for the switching from 
the ON state to the OFF state $\left(\mathbf{S}_{1}\right)$ that from the OFF state to the zero-current state $\left(\mathbf{S}_{2}\right)$, and that for the final switching back to the ON state $\left(\mathbf{S}_{3}\right)$ :

$$
\boldsymbol{\Phi}_{\text {cycle }}=\mathbf{S}_{3} \times \boldsymbol{\Phi}_{i=0} \times \mathbf{S}_{2} \times \boldsymbol{\Phi}_{\text {off }} \times \mathbf{S}_{1} \times \boldsymbol{\Phi}_{\text {on }}
$$

In this case, the state transition matrices $\boldsymbol{\Phi}_{\text {on }}, \boldsymbol{\Phi}_{\text {off }}$ and $\boldsymbol{\Phi}_{i=0}$ are obtained as matrix exponentials as before. In calculating the saltation matrix $\mathbf{S}_{1}$, the switching function is $h_{1}(\mathbf{x}, t)=K_{P}\left(V_{\text {ref }}-x_{1}\right)-$ $v_{\text {ramp }}$, so that the normal is $\mathbf{n}_{1}=\left[-K_{P} 0\right]^{\mathrm{T}}$ and the time derivative is $\partial h_{1} / \partial t=-\left(V_{U}-V_{L}\right) / T$. For calculating $\mathbf{S}_{2}$, the expression for the switching function is simply $h_{2}(\mathbf{x}, t)=x_{2}$. Thus, $\mathbf{n}_{2}=\left[\begin{array}{ll}0 & 1\end{array}\right]^{\mathrm{T}}$ and $\partial h_{2} / \partial t=0$. With these expressions, one can obtain the saltation matrices

$$
\mathbf{S}_{1}=\mathbf{I}+\frac{\left(\mathbf{f}_{\text {off }}-\mathbf{f}_{\text {on }}\right) \mathbf{n}_{1}^{\mathrm{T}}}{\mathbf{n}_{1}^{\mathrm{T}} \mathbf{f}_{\mathrm{on}}+\partial h_{1} / \partial t}, \quad \mathbf{S}_{2}=\mathbf{I}+\frac{\left(\mathbf{f}_{d}-\mathbf{f}_{\mathrm{off}}\right) \mathbf{n}_{2}^{\mathrm{T}}}{\mathbf{n}_{2}^{\mathrm{T}} \mathbf{f}_{\mathrm{off}}+\partial h_{2} / \partial t}
$$

which are evaluated as

$$
\mathbf{S}_{1}=\left[\begin{array}{cc}
1 & 0 \\
\frac{V_{\text {in }}}{L}\left(-\frac{x_{2}}{C}+\frac{x_{1}}{R C}-\frac{V_{U}-V_{L}}{K_{P} T}\right)^{-1} & 1
\end{array}\right], \quad \mathbf{S}_{2}=\left[\begin{array}{cc}
1 & \frac{L x_{2}}{C x_{1}} \\
0 & 0
\end{array}\right]
$$

As mentioned before, $\mathbf{S}_{3}$ is an identity matrix.

To illustrate, we choose the parameter values $L=1 \mathrm{mH}, C=47 \mu \mathrm{F}, T=400 \mu \mathrm{s}, V_{L}=3.8 \mathrm{~V}$, $V_{U}=8.2 \mathrm{~V}, K_{P}=5.0, K_{I}=2.0, V_{\text {ref }}=10 \mathrm{~V}, R=100 \Omega$, and find that at $V_{\text {in }}=18 \mathrm{~V}$ the behavior is stable as shown in Figure 7. The steady-state duty ratios are calculated to be $d_{1}=0.1829$ and $d_{2}=0.3301$ (this was done by simulating the actual system model, including the integrator). Upon calculating the eigenvalues of the monodromy matrix we get the values 0 and -0.758765 . Note that one eigenvalue coming out to be zero is natural since any perturbation in the inductor current goes to zero at the end of the clock period. The other eigenvalue needs to be followed in order to
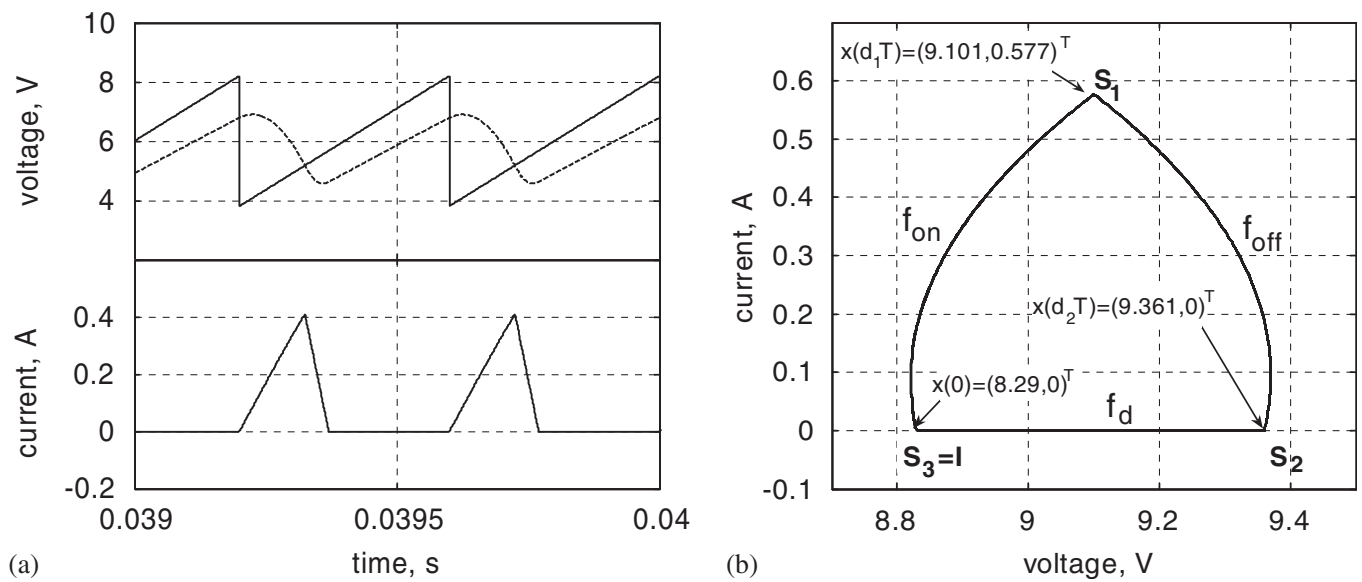

Figure 7. (a) The voltage and current waveforms for the converter in DCM and (b) the orbit in the state space. 
ascertain the system's stability as the external parameters vary. In fact, it is found that subharmonic oscillations set in at $V_{\text {in }}=19.3 \mathrm{~V}$.

\subsection{Pulse width modulation of type-1}

In the case of type-1 PWM (which is more common in digital implementations), one observes the control voltage only at the beginning of a clock period, and the switching signal is generated when that value of the control voltage becomes equal to the ramp voltage (see Figure 2). In this case the complication is that if one writes the switching function as $h(\mathbf{x}, t)=K_{P}\left(V_{\text {ref }}-x_{1}(0)\right)-v_{\text {ramp }}$, one may erroneously conclude that the normal vector is $\left[\begin{array}{ll}0 & 0\end{array}\right]^{\mathrm{T}}$, i.e. it does not exist. The correct way of approaching the problem is to note that switching happens when the state reaches a specific point in the state space, say $\mathbf{x}(t)$, and to express $\mathbf{x}(0)$ in terms of that. Since

$$
\mathbf{x}(t)=\mathrm{e}^{\mathbf{A} t} \mathbf{x}(0)+\int_{0}^{t} \mathrm{e}^{\mathbf{A}(t-\tau)}\left[\begin{array}{c}
0 \\
V_{\text {in }} / L
\end{array}\right] \mathrm{d} \tau
$$

by writing the integral term as $\mathbf{I}(t)$ we can express $\mathbf{x}(0)$ as

$$
\mathbf{x}(0)=\left(\mathrm{e}^{\mathbf{A} t}\right)^{-1}(\mathbf{x}(t)-\mathbf{I}(t))
$$

if $\mathrm{e}^{\mathbf{A} t}$ is invertible. The switching function can now be expressed as

$$
h(\mathbf{x}, t)=\left[\begin{array}{ll}
1 & 0
\end{array}\right]\left(\mathrm{e}^{\mathbf{A} t}\right)^{-1}[\mathbf{x}(t)-\mathbf{I}(t)]-V_{\text {ref }}+v_{\text {ramp }} / K_{P}
$$

To show that this expression really represents the switching function (which is now seen to be dependent on both $x_{1}, x_{2}$ and time), we present in Figure 8 the positions of the above switching function at four different time points within a clock cycle. It is seen that this switching function coincides with the state at $t=d T$.

Having crossed the main hurdle, the rest of the computation proceeds in the same way as before. We take the parameter values $L=20 \mathrm{mH}, C=22 \mu \mathrm{F}, T=110 \mu \mathrm{s}, V_{\text {ref }}=5.2 \mathrm{~V}, R=10.38 \Omega$, $V_{U}=5.5 \mathrm{~V}, V_{L}=0.4 \mathrm{~V}, K_{P}=20$ and $K_{I}=10$. At $V_{\text {in }}=20 \mathrm{~V}$, we obtain the duty ratio $d=0.25997$, and the saltation matrix and the monodromy matrix were obtained as

$$
\mathbf{S}_{1}=\left[\begin{array}{cc}
1 & 0 \\
-0.4885 & 1.5972
\end{array}\right], \quad \boldsymbol{\Phi}_{\text {cycle }}=\left[\begin{array}{ll}
-0.7316 & 3.9498 \\
-0.4328 & 0.9882
\end{array}\right]
$$

The eigenvalues of $\boldsymbol{\Phi}_{\text {cycle }}$ are $0.12834 \pm 0.98496 \mathrm{j}$ with modulus 0.9933 . The system is thus stable, but very close to losing stability.

Whenever the system loses stability with complex conjugate eigenvalues of the complete-cycle state transition matrix, a very special phenomenon occurs. A low-frequency sinusoidal oscillation develops in the output voltage and inductor current waveforms. The experimentally observed waveforms of the control voltage and the inductor current at $V_{\text {in }}=20$ and $19.5 \mathrm{~V}$ are shown in Figure 9. It confirms the observations of the earlier studies on the PWM-1-controlled converters $[28,32,33]$. 



Figure 8 . The position of the switching function and the system trajectory in the state space at four points in time.



(a)

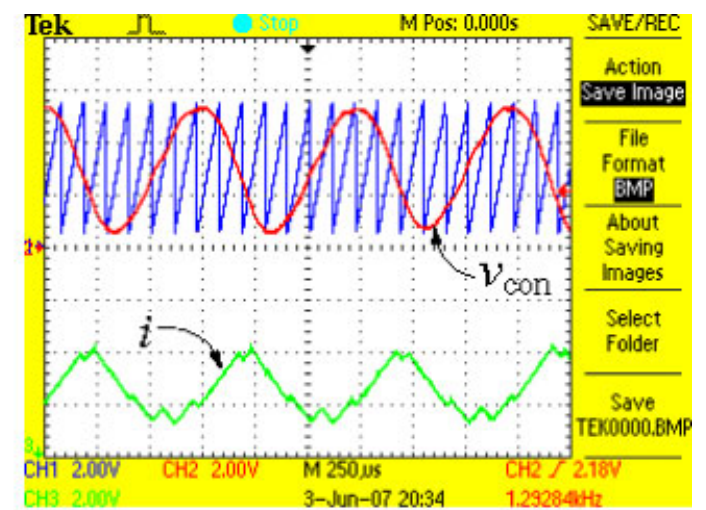

(b)

Figure 9. (a) Stable period-1 orbit at $V_{\text {in }}=20 \mathrm{~V}$ and (b) the slow-scale sinusoidal oscillation at $V_{\text {in }}=19.5 \mathrm{~V}$. 


\section{THE BOOST CONVERTER UNDER CURRENT MODE CONTROL}

Boost converters are generally operated in current mode control to avoid the undesirable transient behavior resulting from the zero in the right-half-plane appearing in the averaged model [7]. There are two types of current mode control strategies. In both, the switch is turned ON at the beginning of every clock period. In the peak current mode control, the maximum value of the inductor current is compared with a reference current signal with a compensation ramp, and the switch is turned OFF when the two become equal. In the average current mode control, the inductor current is compared with a ramp waveform, and the switch is turned OFF when $i<i_{\text {ramp. }}$ It is turned ON again at the next clock instant. We now illustrate the technique of obtaining the state transition matrix over the full cycle for these two control strategies.

\subsection{Peak current mode control}

The peak current-mode-controlled boost converter is shown in Figure 10. The right-hand sides of the state equations are

$$
\mathbf{f}_{\mathrm{off}}=\left[\begin{array}{c}
\frac{x_{2} R-x_{1}}{R C} \\
\frac{V_{\mathrm{in}}-x_{1}}{L}
\end{array}\right], \quad \mathbf{f}_{\mathrm{on}}=\left[\begin{array}{c}
-\frac{x_{1}}{R C} \\
\frac{V_{\mathrm{in}}}{L}
\end{array}\right]
$$

where $x_{1}$ is the capacitor voltage and $x_{2}$ is the inductor current.

Thus, the state matrices for the ON and OFF periods are

$$
\mathbf{A}_{\text {on }}=\left[\begin{array}{cc}
-1 / R C & 0 \\
0 & 0
\end{array}\right], \quad \mathbf{A}_{\text {off }}=\left[\begin{array}{cc}
-1 / R C & 1 / C \\
-1 / L & 0
\end{array}\right]
$$

If the compensation ramp falls $m_{\mathrm{c}}$ amperes in a clock period, the switching condition is $i-\left(I_{\mathrm{ref}}-m_{\mathrm{c}} t / T\right)=0$, and hence the normal vector is given by $\mathbf{n}=\left[\begin{array}{ll}0 & 1\end{array}\right]^{\mathrm{T}}$ and the rate of change

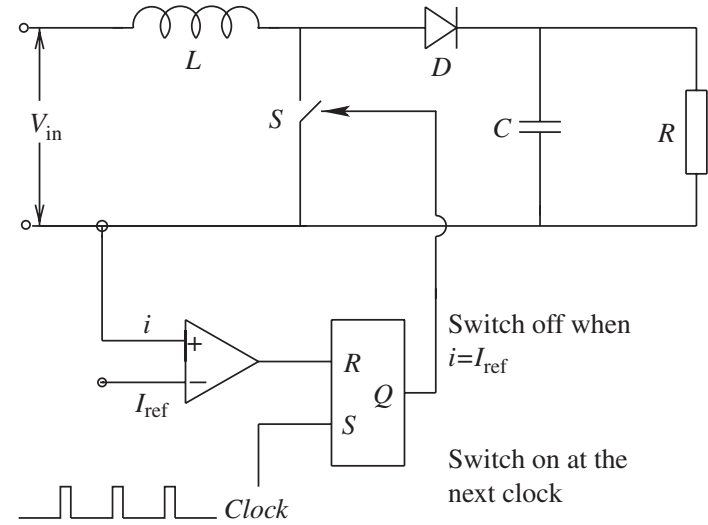

(a)

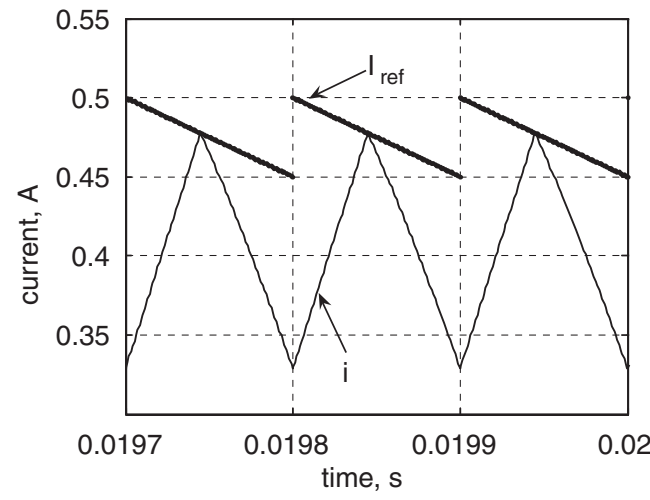

(b)

Figure 10. (a) The peak current-mode-controlled boost converter and (b) the inductor current and reference current waveforms. 
of the switching function $\partial h / \partial t=m_{\mathrm{c}} / T$. Using these, we get the saltation matrix $\mathbf{S}_{1}$ as

$$
\mathbf{S}_{1}=\left[\begin{array}{cc}
1 & \left(\frac{x_{2} R-x_{1}}{R C}+\frac{x_{1}}{R C}\right)\left(\frac{V_{\text {in }}}{L}+\frac{m_{\mathrm{c}}}{T}\right)^{-1} \\
0 & 1+\left(\frac{V_{\text {in }}-x_{1}}{L}-\frac{V_{\text {in }}}{L}\right)\left(\frac{V_{\text {in }}}{L}+\frac{m_{\mathrm{c}}}{T}\right)^{-1}
\end{array}\right]
$$

and $\mathbf{S}_{2}=\mathbf{I}$.

Substituting the values at the switching instant, one can evaluate the exponential matrices and the saltation matrix, from which the monodromy matrix can be obtained as

$$
\boldsymbol{\Phi}_{\text {cycle }}=\mathbf{S}_{2} \times \mathrm{e}^{\mathbf{A}_{\text {off }}(1-d) T} \times \mathbf{S}_{1} \times \mathrm{e}^{\mathbf{A}_{\text {on }} d T}
$$

It is interesting to note that the stabilizing ramp works by influencing the saltation matrix. For example, if we choose the parameters $V_{\text {in }}=4 \mathrm{~V}, I_{\text {ref }}=0.5 \mathrm{~A}, L=1.5 \mathrm{mH}, C=10 \mu \mathrm{F}, R=40 \Omega$, $T=100 \mu$ s and do not use the stabilizing ramp $\left(m_{\mathrm{c}}=0\right)$, the duty ratio is found to be 0.5208 , and the eigenvalues of the monodromy matrix are 0.5725 and -1.2730 , respectively. Since one eigenvalue is outside the unit circle, the orbit is unstable and subharmonic oscillations occur [34,35]. If we set the slope of the compensation ramp $m_{\mathrm{c}}=0.05 \mathrm{~A}$, then the duty ratio is 0.5072 (i.e. still greater than $0.5)$. However, the system becomes stable as the eigenvalues turn out to be 0.5510 and -0.8305 (both inside the unit circle). This happens because the saltation matrix $\mathbf{S}_{1}$ changes

$$
\text { from }\left[\begin{array}{cc}
1 & 18.7500 \\
0 & -0.9358
\end{array}\right] \text { to }\left[\begin{array}{cc}
1 & 14.9886 \\
0 & -0.5876
\end{array}\right]
$$

This provides an alternative explanation of the stabilization of subharmonic oscillations with the compensation ramp.

\subsection{Average current mode control}

The schematic diagram of the average current-mode-controlled boost converter is shown in Figure 11. In this case the $\mathbf{f}_{\text {off }}, \mathbf{f}_{\text {on }}, \mathbf{A}_{\text {off }}$, and $\mathbf{A}_{\text {on }}$ remain the same as in the peak current-modecontrolled boost converter. The switching condition changes to

$$
h(\mathbf{x}, t)=V_{L}+\left(V_{U}-V_{L}\right) \frac{t}{T}-K_{P} x_{2}=0
$$

so that $\mathbf{n}=\left[0-K_{P}\right]^{\mathrm{T}}$ and $\partial h / \partial t=-\left(V_{U}-V_{L}\right) / T$.

Using these, we obtain the saltation matrix

$$
\mathbf{S}_{1}=\left[\begin{array}{cc}
1 & -x_{2} \frac{K_{P}}{C}\left(-\frac{K_{P} V_{\mathrm{in}}}{L}+\frac{-V_{U}+V_{L}}{T}\right)^{-1} \\
0 & 1+x_{1} \frac{K_{P}}{L}\left(-\frac{K_{P} V_{\mathrm{in}}}{L}+\frac{-V_{U}+V_{L}}{T}\right)^{-1}
\end{array}\right]
$$




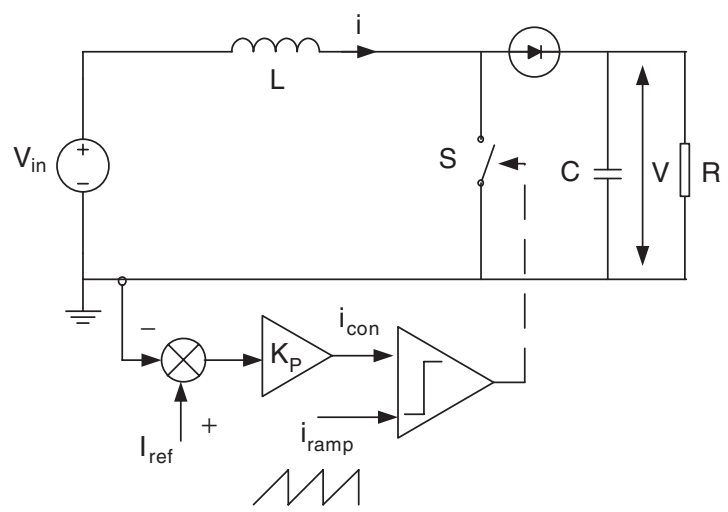

Figure 11. The boost converter with the average current mode control.

For example, if the parameters are $K_{P}=5 \mathrm{~V} / \mathrm{A}, V_{\text {in }}=5 \mathrm{~V}, I_{\text {ref }}=0.8 \mathrm{~A}, L=1.5 \times 10^{-3} \mathrm{H}, C=$ $10 \times 10^{-6} \mathrm{~F}, R=40 \Omega, T=100 \mu \mathrm{s}, V_{L}=0 \mathrm{~V}, V_{U}=2 \mathrm{~V}$, then we obtain

$$
\mathbf{S}_{1}=\left[\begin{array}{ll}
1 & 8.1346 \\
0 & 0.1407
\end{array}\right], \quad \boldsymbol{\Phi}_{\text {cycle }}=\left[\begin{array}{cc}
0.7144 & 7.2322 \\
-0.0264 & -0.1141
\end{array}\right]
$$

whose eigenvalues are $0.3001 \pm 0.1396 \mathrm{j}$ indicating that the system is quite stable as its modulus is much smaller than unity.

\section{INCREASING THE STABILITY MARGIN}

Since the stability of the orbit is given by the state transition matrix over a full clock period, its Equation (2) can be used as a guideline for evolving control strategies. Note that it is composed of two types of matrices: the matrix exponentials and the saltation matrices. The matrix exponentials depend on the state matrix A for the ON and OFF periods (which are constant), and the duty ratio. For constant output voltage, if the input voltage varies, the duty ratio will also vary, and that cannot be controlled. But Equation (2) reveals that there is another handle: the saltation matrix. We show here that the saltation matrix can be manipulated to stabilize the system.

Now, note the expression for the saltation matrix (1). It contains the right-hand sides of the state equations $\mathbf{f}_{\text {on }}$ and $\mathbf{f}_{\text {off }}$ for the ON and OFF periods, which depend on the internal parameters (set by the customer's specifications) and cannot be manipulated. It also depends on the rate of change $\partial h / \partial t$, and the normal vector $\mathbf{n}$, which can be manipulated. This observation provides the theoretical grounding for the proposed control strategies.

\subsection{Strategies for manipulating the saltation matrix}

Let us first focus on the term $\partial h / \partial t$. In the current-mode-controlled converter of Section 4 , this term is directly proportional to $m_{\mathrm{c}}$. The compensation ramp therefore directly affects the saltation matrix (see Equation (10)), and through that the monodromy matrix. This has the effect of pushing the eigenvalues into the unit circle even though the duty ratio exceeds 0.5 . 
In case of the voltage mode control (PWM-2), $\partial h / \partial t=-\left(V_{U}-V_{L}\right) / T$. Since the clock period is effectively constant, the control on the saltation matrix can be exercised through either $V_{U}$ or $V_{L} \cdot{ }^{\S}$ In this paper we illustrate the control through manipulation of the upper tip of the triangular wave which is easily implementable using analog hardware.

Now let us look at the normal vector $\mathbf{n}$. In the case of the voltage mode control, the capacitor voltage $x_{1}$ and the integrator output $x_{3}$ are fed back to decide the control voltage, which is then compared with the ramp. Therefore, the switching surface is parallel to the current axis in the state space (which moves back and forth as the ramp voltage goes up and down). The way to affect the normal vector $\mathbf{n}$ is to change the orientation of this surface by introducing a current feedback to decide $v_{\text {con }}$.

\subsection{Experimental validation}

In the following subsections, we illustrate these possibilities as effective control strategies to avoid subharmonic oscillations.

5.2.1. Voltage-mode-controlled buck converter in PWM-2. We implemented a controller that observes the magnitude of the voltage ripple (which can be used as an indicator of impending onset of instability), and a supervising controller comes into action when the ripple magnitude exceeds a threshold value. The supervising controller notches up the peak value $V_{U}$ of the ramp waveform by $\delta V_{U}$ volts. This increases the slope of the ramp, and hence alters the saltation matrix. The temporal response of the system due to the operation of the supervising controller is shown in Figure 12.

A convenient way of having a panoramic view of the stability status under varying parameter is through what is known as a bifurcation diagram. In obtaining such a diagram one samples a variable (say, the control voltage) at every clock instant. Thus, if the orbit is periodic, one obtains a single point on the CRO screen. When period-2 subharmonic occurs, one gets two points. The variable parameter $V_{\text {in }}$ is fed into channel- 1 and the variable $v_{\text {con }}$ is fed into channel-2 of the CRO, which is put in the $X-Y$ mode. Now, if the input voltage is varied, the point will move in the horizontal direction, and when the subharmonic instability occurs, the point will split into two. The whole process can be captured and stored as image in a digital storage oscilloscope. Figure 13(a) shows the experimentally obtained bifurcation diagram of the converter described in Section 3. It clearly shows that the instability occurs at $V_{\text {in }} \approx 26.7 \mathrm{~V}$.

Suppose we want to extend the range of stable operation to $30 \mathrm{~V}$. By the method given in Section 3, we find that at this input voltage, the eigenvalues are $-1.6619,-0.4089$ and 0.9995 , and hence the system is unstable. By keeping the duty ratio fixed (for a given input voltage)

\footnotetext{
$\S$ We have shown earlier [26] that an alternative way of influencing this term is by adding a small-amplitude sinusoid to the ramp waveform, so that the switching condition becomes

$$
x_{1}-V_{\text {ref }}-V_{\text {ref }} a \sin (\omega t+\phi)-\frac{T V_{L}+\left(V_{U}-V_{L}\right) t}{A T}=0
$$

If one chooses the phase $\phi$ of the sinusoid so that the zero crossing coincides with the nominal steady-state switching instant in each clock cycle, then the introduction of this additional sinusoid has very little influence on the switching instant, and hence on the normal operation of the converter. But since $\partial h / \partial t$ contains the cosine term which attains maximum value at the switching instant, this term is affected. This significantly alters the saltation matrix and the system is stabilized.
} 


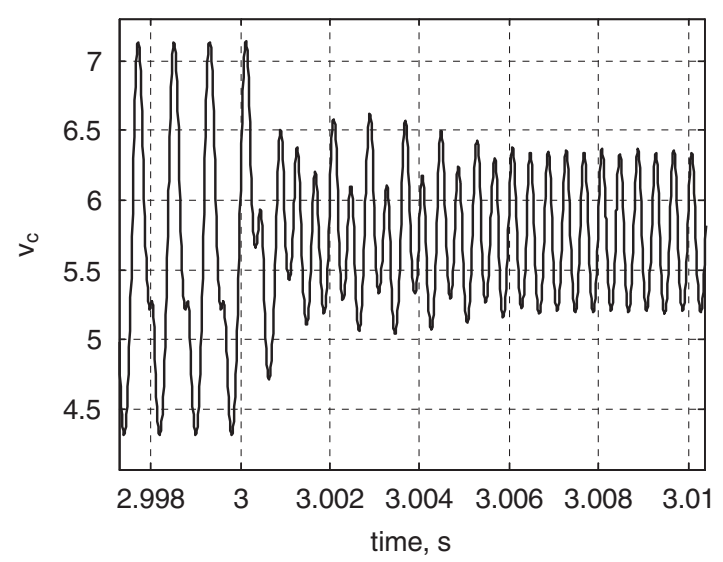

Figure 12. The temporal response showing the action of the supervising controller to stabilize the system at a parameter value where the system is normally unstable.

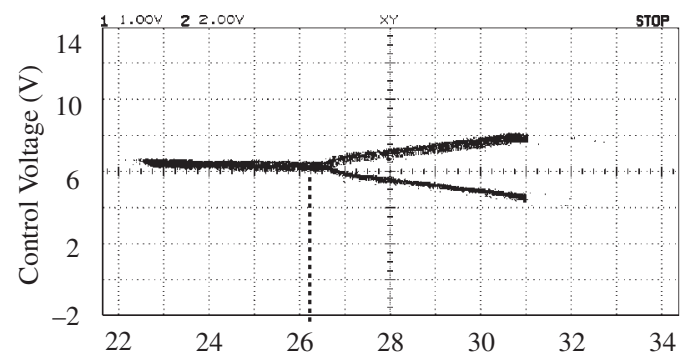

(a)

Input Voltage (V)

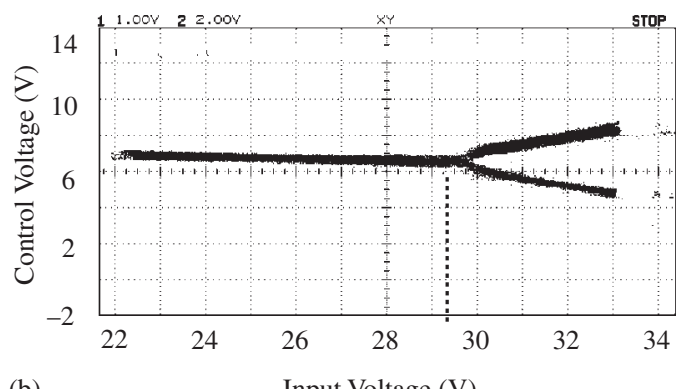

(b)

Input Voltage (V)

Figure 13. The experimentally obtained bifurcation diagram for the voltage-mode-controlled buck converter: (a) under normal PWM-2 control and (b) when the secondary control loop is added with $\delta V_{U}=0.7 \mathrm{~V}$.

we express the monodromy matrix and hence its eigenvalues as a function of $V_{U}$. Then we can solve a nonlinear equation (using the Newton-Raphson method) and that allows us to obtain the required value of $\delta V_{U}$, to optimally design the supervising controller. We find that if the value of $V_{U}$ is pushed up by $1.11 \mathrm{~V}$, then the eigenvalues become $-0.9982,-0.6808$ and 0.9995 , so that the system is stable. In the experiment (Figure 13(b)), we found that $\delta V_{U}=0.7 \mathrm{~V}$ suffices in controlling the system up to $V_{\text {in }}=30 \mathrm{~V}$, because of the small difference in the experimental system and the idealized model.

We have also experimentally verified the stabilization of the system by changing the orientation of the switching surface (by adding current feedback). For the same system, a current feedback path was added so that the control voltage is given by

$$
v_{\mathrm{con}}=K_{P}\left(\frac{v}{k_{1}}+\frac{i}{k_{2}}-V_{\mathrm{ref}}\right)+v_{i}
$$






Figure 14. The experimentally obtained bifurcation diagram for the voltage-mode-controlled buck converter when a current feedback path is added with gain $k_{2}=0.15 \mathrm{~V} / \mathrm{A}$.

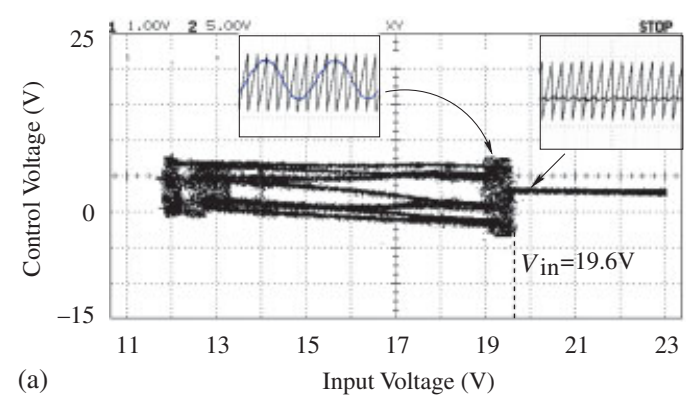

Figure 15. The experimentally obtained bifurcation diagram for the voltage-mode-controlled buck converter: (a) under normal PWM-1 control and (b) when the secondary control loop is added with $\delta V_{U}=0.7 \mathrm{~V}$.

For the experiment, we chose $k_{1}=1$ and $k_{2}=0.15 \mathrm{~A} / \mathrm{V}$, and the resulting bifurcation diagram is shown in Figure 14. By comparing it with the nominal bifurcation diagram of Figure 13(a), it is clear that the current feedback increases the stable range to $31.6 \mathrm{~V}$.

5.2.2. Voltage-mode-controlled buck converter in PWM-1. As we have seen earlier, the common type of instability in PWM-1-controlled buck converter is the sudden onset of slow sinusoidal oscillation, known as quasiperiodicity. In this converter configuration also, we applied the same control technique of increasing the slope of the ramp. The result is shown in the two bifurcations diagrams in Figure 15, one for the standard PWM-1 control and the other for the proposed controller that changes the slope of the ramp. It shows that although the instability occurred at $V_{\text {in }} \approx 19.6 \mathrm{~V}$ for a normal PWM-1-controlled system, the stable range could be extended to $15.5 \mathrm{~V}$ by the proposed control technique. Note that if we allow $V_{U}$ to be varied by a larger extent, the range can be further extended.

\section{CONCLUSIONS}

We have shown that the method developed by Filippov and others for the study of switching mechanical systems is very convenient in studying the fast-scale stability in dc-dc converters. The 
method rests on the computation of the state transition matrix over a complete clock cycle as a product of the state transition matrices for the evolutions through each linear subsystem (the ON state, the OFF state, etc.), and the state transition matrices corresponding to the switching events (the saltation matrices). The eigenvalues of the complete-cycle state transition matrix are the Floquet multipliers, which indicate the stability of the system in fast timescale.

We have applied this technique to a number of converter configurations, namely, the buck converter under voltage mode control (both PWM of types 1 and 2), and the boost converter under peak current mode control and average current mode control. In all these cases we have derived the expressions of the saltation matrices and the state transition matrices for evolution through the linear subsystems-which can be directly used in evaluating the stability margin in converters with similar configurations.

We have shown that the saltation matrix offers a handle in increasing the stability margin. The expression of this matrix shows that it can be manipulated by influencing either the time derivative of the switching function, or its orientation in the state space. In a PWM controller, the rate of change of the switching function can be altered by controlling the slope of the ramp, and its orientation can be controlled by feeding back both voltage and current signals to decide the control voltage. Based on this theoretical premise, we have proposed effective and simply implementable control strategies to increase the range of parameters such as input voltage or load over which the converter remains stable.

\section{ACKNOWLEDGEMENTS}

The authors would like to thank Professor Ralph Kennel (Wuppertal University), Mr Nikolaos Oikonomou (Wuppertal University), Dr David Atkinson (Newcastle University) and Dr Remco Leine (ETH Zurich) for the useful and constructive comments.

\section{REFERENCES}

1. Kassakian JG, Schlecht MF, Verghese GC. Principles of Power Electronics. Addison-Wesley: Reading, MA, 1991.

2. Deane JHB, Hamill DC. Instability, subharmonics, and chaos in power electronics circuits. IEEE Transactions on Power Electronics 1990; 5(3):260-268.

3. Middlebrook RD, Ćuk S. A general unified approach to modeling switching converter power stages. IEEE Power Electronics Specialists' Conference, Cleveland, OH, 1976; 18-34.

4. Krein P, Bentsman J, Bass RM, Lesieutre BC. On the use of averaging for the analysis of power electronic systems. IEEE Transactions on Power Electronics 1990; 5(2):182-190.

5. Verghese GC, Elbuluk ME, Kassakian JG. A general approach to sampled-data modeling for power electronic circuits. IEEE Transactions on Power Electronics 1986; PE-1(2):76-89.

6. Banerjee S, Verghese GC (eds). Nonlinear Phenomena in Power Electronics: Attractors, Bifurcations, Chaos, and Nonlinear Control. IEEE Press: New York, 2001.

7. Tse CK. Complex Behavior of Switching Power Converters. CRC Press: Boca Raton, FL, U.S.A., 2003.

8. Bernardo MD, Garofalo F, Glielmo L, Vasca F. Switchings, bifurcations and chaos in DC-DC converters. IEEE Transactions on Circuits and Systems I 1998; 45(2):133-141.

9. di Bernardo M, Vasca F. Discrete-time maps for the analysis of bifurcations and chaos in DC/DC converters. IEEE Transactions on Circuits and Systems I 2000; 47(2):130-143.

10. Hiskens IA, Pai MA. Trajectory sensitivity analysis of hybrid systems. IEEE Transactions on Circuits and Systems I 2000; 47:204-220.

11. Dranga O, Buti B, Nagy I, Funato H. Stability analysis of nonlinear power electronic systems utilizing periodicity and introducing auxiliary state vector. IEEE Transactions on Circuits and Systems I 2005; 52(1):168-178. 
12. Basak B, Parui S. Incompleteness of bifurcation diagram in predicting the behaviour of Ćuk converter in discontinuous conduction mode. International Journal of Circuit Theory and Applications 2008; 36(4):387-396.

13. Zou J, Ma X, Tse CK, Dai D. Fast-scale bifurcation in power-factor-correction buck-boost converters and effects of incompatible periodicities. International Journal of Circuit Theory and Applications 2006; 34(3):251-264.

14. Wu X, Tse CK, Wong SC, Lu J. Fast-scale bifurcation in single-stage PFC power supplies operating with DCM boost stage and CCM forward stage. International Journal of Circuit Theory and Applications 2006; 34(3):341-355.

15. Huang Y, Iu HHC, Tse CK. Boundaries between fast- and slow-scale bifurcations in parallel-connected buck converters. International Journal of Circuit Theory and Applications 2008; DOI: 10.1002/cta.454.

16. Guesmi K, Hamzaoui A, Zaytoon J. Control of nonlinear phenomena in dc-dc converters: fuzzy logic approach. International Journal of Circuit Theory and Applications 2008; DOI: 10.1002/cta.468.

17. Floquet G. Sur les équations differentielles linéaires à coefficients périodiques. Annales Scientifiques de l'École Normale Supérieure 1883; 12(2):47.

18. Aizerman MA, Gantmakher FR. On the stability of periodic motions. Journal of Applied Mathematics and Mechanics 1958; 22:1065-1078 (translated from Russian).

19. Filippov AF. Differential equations with discontinuous right-hand side. American Mathematical Society Translations 1978; 42(2):199-231.

20. Giaouris D, Banerjee S, Zahawi B, Pickert V. Stability analysis of the continuous conduction mode buck converter via Filippov's method. IEEE Transactions on Circuits and Systems I 2008; 55(4):1084-1096.

21. Kuznetsov YA. Elements of Applied Bifurcation Theory. Springer: New York, NY, U.S.A., 2004.

22. Leine RI, Nijmeijer H. Dynamics and Bifurcations in Non-smooth Mechanical Systems. Springer: Berlin, 2004.

23. Zhusubaliyev ZT, Soukhoterin EA, Rudakov VN, Kolokolov YV, Mosekilde E. Bifurcations and chaotic oscillations in an automatic control relay system with hysteresis. International Journal of Bifurcation and Chaos 2001; 11(5):1193-1231.

24. Zhusubaliyev ZT, Soukhoterin EA, Mosekilde E. Border collision bifurcations and chaotic oscillations in a piecewise smooth dynamical system. International Journal of Bifurcation and Chaos 2001; 11(12):2977-3001.

25. Filippov AF. Differential Equations with Discontinuous Right-hand Sides. Kluwer Academic Publishers: Dordrecht, 1988.

26. Giaouris D, Elbkosh A, Banerjee S, Zahawi B, Pickert V. Stability of switching circuits using complete-cycle solution matrices. International Conference on Industrial Technology, ICIT 2006, Mumbai, India, 2006.

27. Giaouris D, Banerjee S, Zahawi B, Pickert V. Control of fast scale bifurcations in power factor correction converter. IEEE Transactions on Circuits and Systems II 2007; 54(9):805-809.

28. di Bernardo M, Garofalo F, Glielmo L, Vasca F. Quasi-periodic behaviors in dc/dc converters. IEEE Power Electronics Specialists' Conference, Baveno, Italy, 1996; 1376-1381.

29. Papafotiou GA, Margaris NI. Calculation and stability investigation of periodic steady states of the voltage controlled buck DC-DC converter. IEEE Transactions on Power Electronics 2004; 19(4):959-970.

30. Fossas E, Olivar G. Study of chaos in the buck converter. IEEE Transactions on Circuits and Systems I 1996; 43(1):13-25.

31. Tse CK. Flip bifurcation and chaos in three-state boost switching regulators. IEEE Transactions on Circuits and Systems I 1994; 41(1):16-23.

32. Aroudi AE, Leyva R. Quasi-periodic route to chaos in a PWM voltage-controlled dc-dc boost converter. IEEE Transactions on Circuits and Systems I 2001; 48(8):967-978.

33. Aroudi AE, Benadero L, Toribio E, Machiche S. Quasiperiodicity and chaos in the dc-dc buck-boost converter. International Journal of Bifurcation and Chaos 2000; 10(2):359-371.

34. Chan WCY, Tse CK. Study of bifurcations in current programmed dc/dc boost converters: from quasiperiodicity to period doubling. IEEE Transactions on Circuits and Systems I 1997; 44(12):1129-1142.

35. Banerjee S, Chakrabarty K. Nonlinear modeling and bifurcations in the boost converter. IEEE Transactions on Power Electronics 1998; 13(2):252-260. 